Ann. Biol. anim. Bioch. Biophys., 1974, 14 (4-A), 633-650.

\title{
CINÉTIQUE FOLLICULAIRE ET MATURATION OVOCYTAIRE CHEZ LE VEAU TRAITÉ PAR L'ACÉTATE DE FLUOROGESTONE ET LA PMSG
}

\author{
J. ARRAU (1) \\ avec la collaboration technique de P. CHESNE \\ Station de Physiologie animale, \\ Centre national de Recherches zootechniques, I. N. R. A., \\ 78350 Jouy en Josas

\section{RÉSUMÉ}

Nous avons étudié la croissance folliculaire préovulatoire et la maturation ovocytaire chez quatre groupes de veaux impubères : - groupe témoin (aucun traitement) - groupe de veaux qui ont reçu pendant 4 jours de l'acétate de fluorogestone (FGA) libéré à partir d'une éponge vaginale - deux groupes de veaux qui ont reçu en outre le jour de la mise en place de l'éponge, une injection intramusculaire de PMSG (I 800 UI). Pour le groupe non traité, 35 p. Ioo de la population folliculaire totale des deux ovaires contient des ovocytes à l'état dictyé, dont seulement $36 \mathrm{p}$. Ioo appartiennent à des follicules complètement normaux; le reste de la population folliculaire (64 p. roo) contient des ovocytes en reprise de méỉose. Pour le groupe ayant reçu le progestagène, $74 \mathrm{p}$. Ioo de la population folliculaire totale présente des ovocytes à l'état dictyé, dont 62 p. Ioo sont contenus dans des follicules complètement normaux; le reste de la population folliculaire (26 p. I0o) contient des ovocytes en début de reprise de la méíose.

Ces résultats montrent que le FGA diminue la fréquence des follicules atrétiques ; il empêche simultanément la reprise spontanée de la méiose.

Pour le groupe recevant de la PMSG + FGA, on peut voir que l'effet favorable du FGA, quand il est donné seul, paraît contrebalancé par la stimulation gonadotrope apportée par la PMSG. Nous avons déterminé aussi à quel moment du traitement on peut trouver les follicules, préovulatoires chez ce dernier groupe de veaux traités. Ils sont présents 40 heures après le retrait de l'éponge; leur diamètre varie entre I 2 et $14 \mathrm{~mm}$ et l'ovocyte qu'ils contiennent se trouve presque toujours en métaphase II (MII).

(1) Adresse actuelle : Instituto de Ciencias Biologicas, Lab. Embriología, Universidad Católica, Casilla II4-D, Santiago (Chile). 


\section{INTRODUCTION}

L'atrésie est la destinée normale de la plupart des follicules et des ovocytes, et, à tout moment de la vie, on trouve dans l'ovaire une proportion élevée de follicules atrétiques. Après traitement gonadotrope, la production d'ovocytes normaux est donc tributaire du nombre de follicules normaux susceptibles de répondre à cette stimulation exogène.

Chez tous les Mammifères étudiés, il existe une période prépubertaire pendant laquelle l'ovaire présente un nombre élevé de follicules à antrum et on sait que 1'injection de gonadotropines provoque le plus fort taux d'ovulation qu'il est possible d'obtenir pour une espèce donnée (PICON, I956; Zarrow et WIIsoN, I96I, chez le Rat. Gate, Runner et Jackson, I957 ; Zarrow et Wilson, I96I, chez la Soutis. Fox, Cavanaugh et Zarrow, I954, chez la Lapine. Avery, Fahning et Graham, I962; HAFEz, Sugie et Hunt, I963, chez le Veau).

Désirant obtenir à des fins expérimentales ou appliquées des ovocytes de Bovins, plusieurs auteurs ont tenté d'obtenir une superovulation chez le Veau de 3 à 5 mois, avec des fortunes diverses et toujours un taux de fécondation faible (AvERY, FAHning et Graham, I962 ; Hafez, Sugie et Hunt, I963; Testart, I972 $a, b$ ).

Nous avons cherché à connaître quel était l'état réel de la population folliculaire et ovocytaire chez le Veau normal, après freinage de l'activité gonadotrope ou après stimulation par PMSG, ceci afin de définir les modalités d'un traitement hormonal capable d'induire une superovulation importante d'ovocytes.

Les ovaires de Bovins impubères répondent à l'injection de gonadotropines depuis leur naissance (CASIDA et al., I943; Rowson, I95I ; MARDEN, I952-I953), donc plus précocement que la plupart des Mammifères.

L'administration de PMSG ou de FSH, suivie d'une injection ovulante de HCG (Hafez, Sugie et Hunt, I963; Iineweaver et Hafez, I970; Hafez, I970 ; TesTART, I972 a) ou de LH (AVERY, FAHNING et GRAHAM, I962 ; ONUma et al., I969; Onuma, Hahn et Foote, I970; Seider, Larson et FoOTE, I97I), provoque des taux d'ovulation variables d'un individu à l'autre.

TESTART (I972 $a, b)$ a proposé, chez le Veau recevant une injection de PMSG, l'usage simultané d'un progestagène de synthèse le FGA (1), pour inhiber une décharge endogène prématurée de $\mathrm{L}_{4} \mathrm{H}$. I5 à 20 heures environ après l'arrêt d'un traitement court de 4 jours de FGA, une décharge de $\mathrm{L}_{4} \mathrm{H}$ se produit (TESTART et KanN, I973), susceptible d'entraîner des ovulations qui, chronologiquement, sont mieux groupées qu'en l'absence du progestagène.

L'existence de cette décharge endogène de $\mathrm{L} H$ dispense d'une injection d'HCG pour obtenir la superovulation. L'ovulation commence environ 40 heures après le retrait du FGA, c'est-à-dire 24 heures après le pic maximal de décharge de I.H (TESTART, I972 a), données s'accordant avec celles observées chez la Vache cyclique (Henricks, Dickey et Niswender, I970 ; Dobson, Hopkins et Word, I973).

(1) Acétate de Fluorogestone (I 7- $\alpha$-acétoxy- 9- $\alpha$-fluoro- I I- $\beta$-hydroxypregn-4-en-3,20 dione). 


\section{MATÉRIEL E'T MÉTHODES}

\section{I. - Animaux utilisés et traitements}

Nous avons utilisé 52 veaux de race Française Frisonne Pie Noire (FFPN) âgés de 3 mois environ (poids : $\mathrm{I} 3 \mathrm{I} \pm \mathrm{I}, 5 \mathrm{~kg}$ ) et nourris exclusivement au lait artificiel.

On a constitué 4 groupes :

Groupe $A$ : 3 veaux témoins ne subissent aucun traitement. Ils ont été choisis au hasard à deux mois d'intervalle, a fin d'éviter l'influence éventuelle d'un facteur saisonnier ou d'un facteur commun dû à l'origine des animaux.

Groupe $B: 2$ veaux ont reçu pendant 4 jours de l'acétate de fluorogestone (FGA-Searle) libéré à partir d'une éponge vaginale de polyuréthane imprégnée de $60 \mathrm{mg}$ de FGA (TESTART, I972a).

Groupe $C: 3$ autres veaux ont reçu en outre le jour de la mise en place de l'éponge, une injection intramusculaire de I 800 UI de PMSG (Intervet). L'un des veaux a été abattu 48 heures après le début de ce traitement et les deux autres 72 heures après.

Groupe D: 44 veaux ont été traités de la même manière, mais ils ont été abattus, soit au moment du retrait de l'éponge, soit $4,5, \mathbf{I} 6,21,26,40,48,72$ et 96 heures après.

Le tractus et les ovaires sont prélevés à l'abattage qui a lieu au laboratoire. Les ovaires et l'utérus sont pesés.

Les follicules visibles à la surface de l'ovaire sont dénombrés et mesurés à l'aide d'une échelle millimétrique avant fixation, soit de l'ovaire entier, soit des ovocytes prélevés par aspiration. Le délai entre l'abattage de l'animal et la fixation de tous les ovocytes n'excède jamais 30 minutes et pour les ovaires fixés en entier, i 5 minutes.

$$
\text { II. - Origine des ovocytes étudiés }
$$

Une partie des ovocytes des groupes C et D a été obtenue par aspiration dans des follicules de différentes tailles à l'aide d'une pipette en verre, à température ambiante. Après rinçage dans une solution de citrate de sodium à $3,5 \mathrm{p}$. I oo (pression osmotique 280 milliosmoles), puis dans une solution physiologique de Locke, les ovocytes sont fixés dans le mélange de Bouin-Hollande pendant un minimum de 24 heures.

L'autre partie des ovocytes, provenant des groupes A et B en totalité, C et D partiellement, ont été étudiés in situ dans leurs follicules. Les ovaires sont fendus longitudinalement au niveau du hile pour augmenter la surface de contact avec le fixateur et immergés dans la solution de Bouin-Hollande. Le volume de solution utilisé est 3 à 4 fois plus grand que celui de la masse ovarienne. La fixation dure 3 à 4 semaines, sous vide pendant les 12 premières heures, pour éviter une rétraction folliculaire toujours visible dans les images de RAJAkoski (I960). Les ovaires sont lavés à l'eau distillée pendant 8 heures. On procède alors à la séparation des follicules qui sont à la surface de l'ovaire à l'aide de ciseaux fins. Ils sont groupés par tailles et on en prend au hasard un nombre variable de chaque groupe pour une étude micromorphologique.

\section{III. -.. Techniques histologiques}

a) Les ovocytes isolés, prélevés dans les follicules, sont inclus par la technique de double inclusion gélose-paraplaste (Thibault, I949). Ils sont coupés à Io $\mu$ d'épaisseur, puis colorés à l'hématoxyline de Regaud et à l'éosine.

b) Les follicules sont coupés à ro $\mu$ d'épaisseur et colorés, soit à l'hématoxyline de Regaud et à l'éosine, soit au trichrome de Masson, soit à l'APS (acide périodique-Schiff) pour bien mettre en évidence la membrane basale.

$$
\text { IV. - Observations }
$$

\section{Pour les follicules.}

Chaque follicule plus petit que $3 \mathrm{~mm}$ environ est mesuré après coloration à l'aide d'un micromètre oculaire au niveau de la coupe où l'ovocyte montre le matériel nucléaire. Le diamètre moyen 
est estimé égal à la demi-somme des deux plus grands diamètres perpendiculaires, intérieurs à la thèque interne (ChOUdARY, Gier et Marion, I968).

Pour reconnaître les altérations au niveau des structures folliculaires, on a pris en considération la fréquence des mitoses, des cellules pycnotiques, des corps atrétiques et de la lutéinisation, l'importance du décollement de la granulosa par rapport à la thèque interne, ainsi que l'existence d'altérations plus discrètes au niveau de la membrane basale. Nous avons également tenu compte du degré d'isolement de l'ovocyte et de son cumulus par rapport à la granulosa.

\section{Pour l'ovocyte.}

Nous nous sommes attaché seulement à l'étude de la reprise de la méiose et nous utiliserons le terme de maturation ovocytaire pour qualifier ce processus de transformation nucléaire.

Pour l'étude de la dégénérescence de l'ovocyte ou des structures qui lui sont liées, nous avons considéré : l'état nucléaire, l'homogénéité du cytoplasme (Moss, Wrenn et Sykes, I954; RaJAKoskI, I960), la forme et l'union des cellules de la corona radiata et la lutéinisation de ces cellules.

Pour l'état nucléaire, nous avons distingué entre le stade dictyé et la première métaphase, un stade prométaphase caractérisé par l'apparition des chromosomes et la disparition progressive du nucléole, la membrane nucléaire demcurant distincte.

\section{RÉSUL,TATS E'T DISCUSSION}

\section{A. -- Poids des ovaires}

a) Veaux témoins et veaux traités seulement avec FGA pendant 4 jours (Groupes A et B).

Le tableau I montre que le poids de l'ovaire n'est pas affecté par le traitement avec le FGA. Le poids des ovaires des veaux des groupes $A$ et $B$ ne diffère pas : $3,7 \pm 0,25$ contre $3,35 \pm \mathrm{I}, \mathrm{O}$. Il est semblable au poids des ovaires mentionné par DESJARDins et HAFS (I969) pour des veaux de même âge (Pl. I, photo I.).

\section{TABLEAU I}

Poids moyen des ovaires (en grammes, rapporté à Ioo $\mathrm{kg}$ de poids vif) et nombre moyen de follicules estimés par examen des ovaires in toto

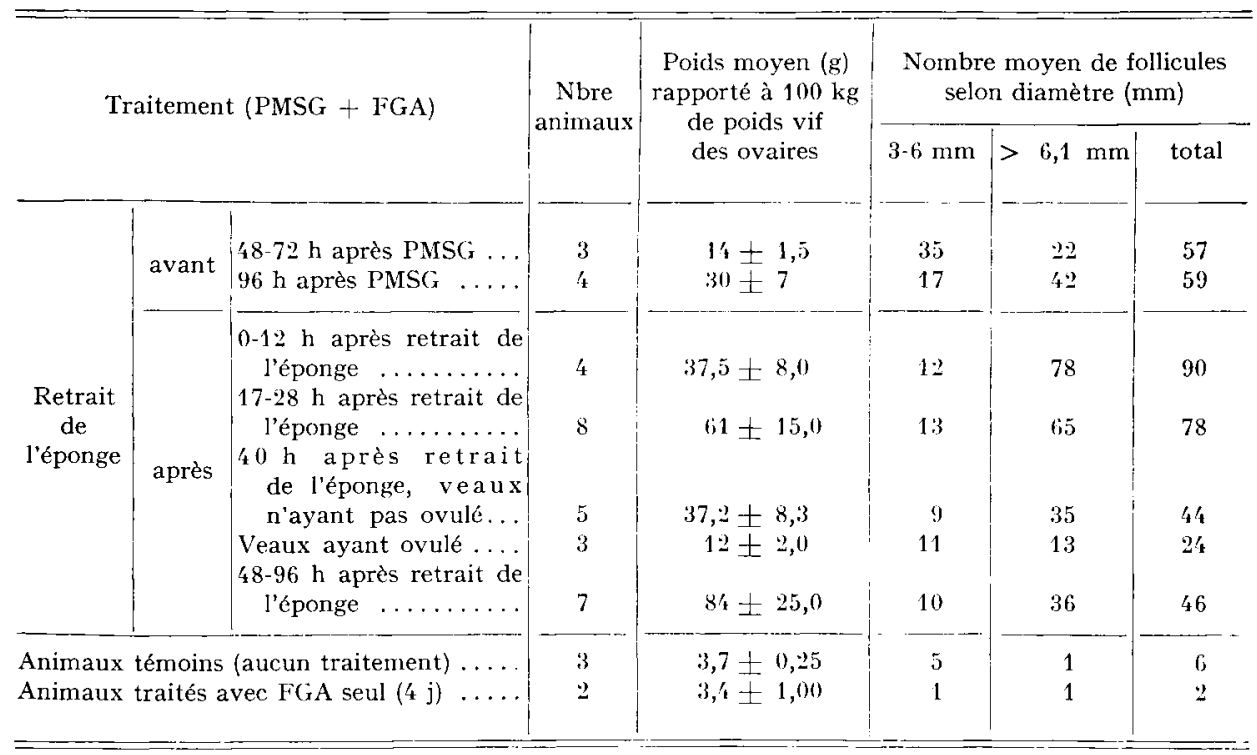


b) Veaux traités avec PMSG $+F G A$ (Groupes C et D).

Le poids de l'ovaire croît assez régulièrement pendant les 96 heures qui suivent le début du traitement, passant de $3,5 \mathrm{~g}$ à $30 \mathrm{~g}$. Après le retrait de l'éponge, sa croissance est plus rapide; il a redoublé de poids en 26 heures ( $67 \pm 7 \mathrm{~g})$; au moment de l'ovulation, le poids de 1'ovaire a régressé jusqu'à I2 $22 \mathrm{~g}$ chez les animaux dont l'ovulation a commencé, mais aussi chez les veaux qui n'ont pas ovulé, probablement par suite d'une atrésie importante (tabl. I ; P1. I, photos 2, 3, 4).

\section{B. - Étude histologique du follicule et de son ovocyte}

a) Présence des corps de Call et Exner.

25 p. Ioo des follicules provenant de veaux traités seulement au FGA présentaient plusieurs corps de Call et Exner. Ces corps sont présents aussi bien dans la granulosa que dans le cumulus ovigère. Chez les veaux traités par PMSG $+\mathrm{FGA}$, on ne les a observés que dans 2 follicules sur 30 étudiés pour un même veau. Nous n'avons pas considéré cette structure comme un indice de dégénérescence, contrairement à MARION et GIER (I97I), à cause de la grande contradiction qui existe dans la littérature sur ses origines et fonctions (MOTTA, I965).

b) Étude de follicules provenant d'ovaires de veaux traités avec PMSG $+F G A$.

I. Follicules avant la décharge ovulante (P1. II, photos 5, 6 et 7).

L,eurs diamètres varient de 0,4 à Io $\mathrm{mm}$. L'ovocyte, au stade dictyé ou de pro métaphase a un cytoplasme homogène ou contient des granules assez éosinophiles. Le cumulus oophorus est constitué de cellules polyédriques, à noyau plus ou moins vésiculeux et à cytoplasme éosinophile. L'ensemble de ces cellules se trouve le plus souvent en continuité complète avec la granulosa.

Une membrane basale qui sépare la thèque interne de la granulosa est bien visible (P1. II, photos 5 et 7 ). Avec la croissance du follicule, la forme des cellules contiguës à cette membrane change; d'abord allongées elles deviennent cubiques, puis polyédriques. Les cellules bordant la cavité folliculaire sont toujours polyédriques.

2. Follicules préovulatoires (P1. III, photos II $a, b, c, \mathrm{I} 2$ et I3).

Leurs diamètres varient de I2 à I4 mm. L'ovocyte (P1. III, photos II $a, b, c)$ possède un cytoplasme très homogène. Son noyau, quelquefois au stade MI, est le plus souvent au stade MII.

Avant l'ovulation, l'ovocyte est totalement libre dans l'antrum, seulement entouré des cellules de la corona retenues à l'ovocyte par de fins prolongements cytoplasmiques qui contribuent à leur donner une forme allongée.

I,e cumulus oophorus n'est plus représenté que par des cellules séparées les unes des autres, baignant dans le liquide folliculaire secondaire (P1. III, photo II $a$ ). L'activité mitotique est nulle. Un nombre appréciable de cellules pycnotiques sont présentes, surtout dans la zone de contact entre le cumulus et la granulosa.

Dans tous les follicules observés, la membrane basale a presque totalement disparu (P1. III, photos I2 et I3). Elle ne se décolle jamais de la thèque interne. Sa disparition débute dans les parties les plus éloignées du cumulus et s'en rapproche progressivement. 
Là oì elle disparaît, les cellules de la granulosa gonflent, devenant sphériques et hyperchromatiques. Le phénomène commence vers l'antrum où les cellules s'écartent les unes des autres, prenant un aspect en éventail. Leur sécrétion doit contribuer à la formation du liquide folliculaire secondaire.

Au pôle opposé à la macula pellucida, la paroi du follicule forme des replis constitués par les cellules de la granulosa et les cellules de la thèque interne. Cet ensemble est distribué autour d'un ou plusieurs vaisseaux sanguins qui ont atteint un grand développement avant 1'ovulation (P1. III, photo I2).

Comme Priedkalns, Wfber et Zemjanis (I968) l'avaient décrit, nous observons aussi que, dans ces follicules, la lutéinisation des cellules de la granulosa a débuté (P1. III, photo I3).

Ce sont les cellules de la granulosa et de la thèque interne les plus éloignées de l'ovocyte qui se lutéinisent le plus rapidement (P1. III, photo I3).

En outre, de nombreux leucocytes éosinophiles apparaissent, accompagnés de cellules pycnotiques et de corps atrétiques en faible quantité.

\section{Follicules lutéinisés (P1. II, photos 8, 9 et ro).}

La lutéinisation se produit dans tous les follicules grands ou petits, 48 à 96 heures après le retrait de l'éponge. La caractéristique externe principale des grands follicules est une paroi opaque, bien irriguée. L'étude histologique révèle qu'elle est lutéinisée. Il n'y a pas d'activité mitotique et la membrane basale n'est plus visible (P1. II, photo Io). Presque tous les ovocytes inclus dans ces follicules sont en MI (grande masse chromatinienne), sans chromosomes identifiables. Les cellules du cumulus oophorus proches de l'ovocyte forment un ensemble de cellules lutéiniques très serrées (P1. II, photo 9). Les cellules du cumulus oophorus plus éloignées de l'ovocyte, déjà lutéinisées, forment de petits groupes généralement sphériques (P1. II, photos 8 et 9). Ellles présentent très peu d'affinité pour l'hématoxyline et l'éosine, cependant sans signe de dégénérescence. Le liquide folliculaire secondaire est présent seulement dans cette région.

Certains follicules lutéinisés de grande taille sont envahis par des leucocytes (éosinophiles) qui entourent la masse des cellules du cumulus déjà lutéinisées.

4. Follicules en dégénérescence.

Le premier indice de dégénérescence folliculaire consiste en la présence de nombreux corps atrétiques et de cellules pycnotiques.

La plupart de ces follicules sont d'un petit diamètre; ils présentent des ovocytes depuis l'état dictyé jusqu'à la MII. Mais contrairement aux ovocytes ayant repris leur méiose sous l'influence de la décharge ovulante endogène, ces cellules périovocytaires restent cubiques et ne présentent jamais l'image caractéristique de la corona préovulatoire. Quand le follicule contient un ovocyte dictyé, il montre très peu de signes de dégénérescence (quelques corps atrétiques, cellules de la granulosa libres dans le liquide folliculaire, granulosa décollée de la thèque interne). La membrane basale a très rarement disparu.

Dans certains petits follicules, l'ovocyte est complètement libre ou attaché à des cellules de la granulosa, mais toujours entouré de cellules du cumulus, apparemment normales. Dans ce cas, la paroi follictulaire montre soit des signes de dégénérescence de sa granulosa (corps atrétiques et cellules pycnotiques libres dans la cavité folliculaire), soit un aspect apparemment lutéinisé. 
Au niveau de la thèque interne, il est difficile de trouver des signes de dégénérescence ; cependant, dans certains cas, il n'y a plus de cellules thécales, mais seulement du tissu conjonctif, riche en fibres collagènes. Ce fait coïncide généralement avec une diminution très accentuée de la vascularisation.

\section{C. - Population folliculaire}

a) Veaux témoins et veaux traités seulement avec FGA pendant 4 jours (Groupes $\mathrm{A}$ et B).

On trouve davantage de follicules de plus de $3 \mathrm{~mm}$ à la surface de l'ovaire des témoins ( 13 contre 4) mais, au total, un nombre plus grand de follicules de taille $<3 \mathrm{~mm}$ chez les animaux traités (I34 contre 96) (tabl. 2).

\section{TABIEAU 2}

Effectifs folliculaires à antrum et stade nucléaive des ovocytes trouvés sur coupes, chez les animaux témoins ou traités avec FGA seul

\begin{tabular}{|c|c|c|c|c|c|c|c|c|c|}
\hline & \multirow{3}{*}{$\begin{array}{c}\text { Nombre } \\
\text { total } \\
\text { follicules } \\
\grave{a} \text { antrum }\end{array}$} & \multicolumn{8}{|c|}{ Répartition des follicules } \\
\hline & & \multicolumn{4}{|c|}{$0,2-1 \mathrm{~mm}$} & \multicolumn{4}{|c|}{$1,1-3 \mathrm{~mm}$} \\
\hline & & $\begin{array}{l}\text { Nbre } \\
\text { foll. }\end{array}$ & $\begin{array}{l}\text { Dic- } \\
\text { tyé }\end{array}$ & PM & $\underset{I-I I}{M}$ & $\begin{array}{l}\text { Nbre } \\
\text { foll. }\end{array}$ & $\begin{array}{l}\text { Dic- } \\
\text { tyé }\end{array}$ & PM & $\stackrel{M}{\mathrm{I}-\mathrm{II}}$ \\
\hline $\begin{array}{c}\text { Veaux non traités } \\
6405-002\end{array}$ & 109 & 46 & 31 & 9 & 6 & 50 & 8 & 36 & 6 \\
\hline \multirow[t]{4}{*}{$\begin{array}{l}\text { Veaux traités avec FGA } \\
04^{\prime}-6856\end{array}$} & 138 & 68 & 59 & 5 & 4 & 66 & 43 & 18 & 5 \\
\hline & \multirow{3}{*}{$\begin{array}{c}\text { Nombre } \\
\text { total } \\
\text { follicules } \\
\text { à antrum }\end{array}$} & \multicolumn{8}{|c|}{ Répartition des follicules } \\
\hline & & \multicolumn{4}{|c|}{$3,1-6 \mathrm{~mm}$} & \multicolumn{4}{|c|}{$>6 \mathrm{~mm}$} \\
\hline & & $\begin{array}{l}\text { Nbre } \\
\text { foll. }\end{array}$ & $\begin{array}{l}\text { Dic- } \\
\text { tyé }\end{array}$ & PM & $\begin{array}{c}M \\
I-I I\end{array}$ & $\begin{array}{l}\text { Nbre } \\
\text { foll. }\end{array}$ & $\begin{array}{l}\text { Dic- } \\
\text { tyé }\end{array}$ & PM & $\underset{I-I I}{M}$ \\
\hline $\begin{array}{l}\text { Veaux non traités } \\
66^{\prime} 105-002\end{array}$ & 109 & 11 & 0 & 9 & 2 & 2 & 0 & 2 & 0 \\
\hline $\begin{array}{c}\text { Veaux traités avec } \mathrm{FGA} \\
0^{\prime} 4^{4}-6856\end{array}$ & 138 & 2 & 1 & 1 & 0 & 2 & 1 & 1 & 0 \\
\hline
\end{tabular}

b) Veaux traités avec PMSG $+F G A$ (Groupes $\mathrm{C}$ et $\mathrm{D}$ ).

Le nombre de follicules présents à la surface des deux ovaires augmente régulièrement après le début du traitement, passant de 13 à 70 environ au moment du retrait de l'éponge. Dans les heures qui suivent, ce nombre augmente encore et parfois dépasse la centaine. Puis, après l'ovulation, il se rapproche du nombre trouvé avant 
le traitement. Il convient de remarquer que le nombre de follicules visibles diminue même chez les animaux n'ovulant pas, ce qui confirme l'hypothèse que l'atrésie est responsable de la réduction du poids de l'ovaire, même sans ovulation (tabl. I).

\section{D. - Maturation ovocytaire et croissance folliculaire}

Cette étude a été réalisée sur 870 ovocytes au total, dont 350 collectés par aspiration de follicules dont le diamètre était estimé sur l'ovaire in toto, et 520 ovocytes étudiés après fixation du follicule entier, leur diamètre étant déterminé sur coupes histologiques.

a) Ovocytes provenant de veaux témoins et de veaux traités avec le FGA pendant 4 jours (Groupes A et B).

Chez les veaux témoins, 35 p. Ioo de la population folliculaire totale des deux ovaires contient des ovocytes à l'état dictyé, dont seulement $36 \mathrm{p}$. Ioo appartiennent à des follicules complètement normaux ; le reste de la population folliculaire (64 p. IOo)

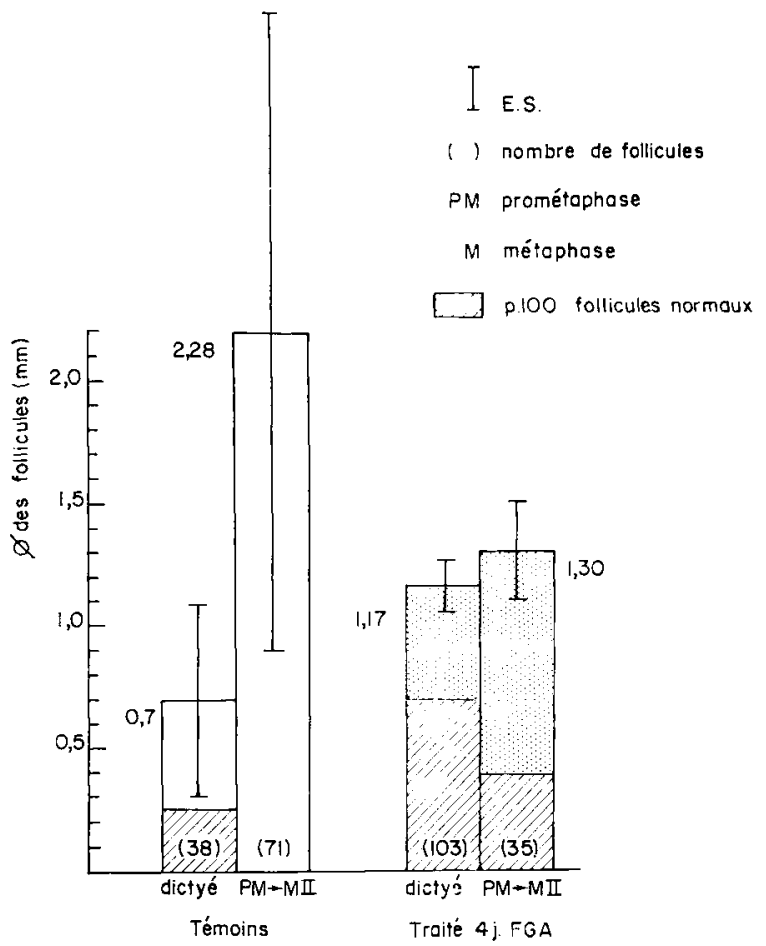

FIG. I. - Répartition de la population folliculaire (nombre et diamètre moyen des follicules à antrum) en fonction des stades du noyau ovocytaire chez les animaux non traités avec PMSG (tabl. 3)

contient des ovocytes en reprise de méiose, dont tous les follicules présentent des signes de dégénérescence. Il n'y a donc que I4 ovocytes dictyés dans des follicules à antrum sains (tabl. $3 a$, fig. I), sur les deux ovaires d'un veau normal. 
Chez les veaux ayant reçu le progestagène, la situation est différente : $74 \mathrm{p}$. roo de la population folliculaire totale présente des ovocytes à l'état dictyé, dont 62 p. Ioo sont contenus dans des follicules complètement normaux; le reste de la population folliculaire (26 p. Ioo) contient des ovocytes présentant un début de reprise de la méiose ; de ceux-ci, 42 p. Ioo étaient dans des follicules normaux (tabl. $3 b$, fig. I). Ces résultats montrent que l'acétate de fluorogestone diminue la fréquence des follicules atrétiques et empêche simultanément la reprise spontanée de la méiose. On trouve en effet 64 ovocytes dictyés contenus ans des follicules normaux, soit 5 fois plus que chez le Veau non traité. Enfin, le nombre total de follicules est plus grand, I38 contre Iog (tabl. 2, fig. I) : les résultats ici trouvés confirment l'hypothèse que nous avions émise (TESTART et ARRAU, I973) : le FGA empêche l'atrésie folliculaire.

La taille des follicules est très variable chez les veaux témoins, beaucoup plus homogène chez les veaux traités, en particulier pour les follicules contenant des ovocytes à noyau dictyé.

Chez les témoins, la taille moyenne des follicules avec ovocyte dictyé est de $0,72 \pm 0,4 \mathrm{~mm}$. Chez les traités elle est de I,I $7 \pm 0, \mathrm{I} \mathrm{mm}$.

\section{TABLEAU 3}

Effectifs et caractéristiques des follicules selon le stade nucléaire de l'ovocyte chez les animaux témoins ou traités avec FGA seul

3 a. - Veaux non traités (numéros 6405 et 002 )

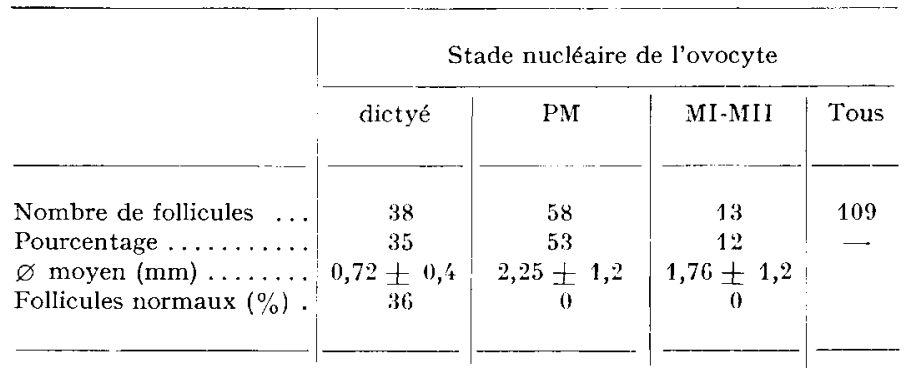

3 b. - Veaux traités avec FGA (numéros 044 et 6856)

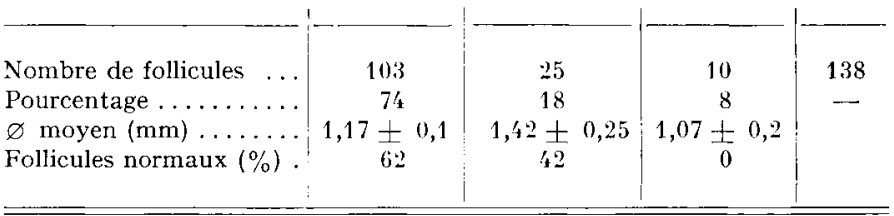

b) Ovocytes provenant de veaux traités avec $P M S G+F G A$ (Groupes $\mathrm{C}$ et $\mathrm{D})$.

On constate que, 48 à 72 heures après le début du traitement, le pourcentage de follicules normaux contenant des ovocytes au stade dictyé est le même que chez les veaux témoins.

L'effet favorable du FGA qui prévient l'évolution atrétique quand il est donné seul (tabl. 3 b), paraît contrebalancé par la stimulation gonadotrope apportée par la PMSG. 
La comparaison des pourcentages des ovocytes à l'état dictyé et des ovocytes ayant repris leur méïose (dans la majorité des cas par suite de la dégénérescence folliculaire) confirme l'effet défavorable de la PMSG. En effet, le rapport ovocytes dictyés/ovocytes en prométaphase + métaphase est de :

0,54 pour les veaux témoins ;

0,54 pour les veaux recevant FGA + PMSG et examinés 48 à 72 heures après le début du traitement;

3,0 pour les veaux recevant le FG seul et examinés au moment du retrait de l'éponge.

Il en résulte que le nombre de follicules qui pourraient être disponibles par suite de l'action favorable du FGA pour répondre à une décharge endogène de $\mathrm{L}_{\mathrm{L}} \mathrm{H}$, est réduit par la PMSG. Cependant, la taille des follicules contenant des ovocytes au stade dictyé à la fin du traitement par le FGA seul, est en moyenne de $\mathrm{I}, \mathrm{I} 7 \mathrm{~mm}$ (fig. I), tandis que $40 \mathrm{p}$. Ioo dépassent $6 \mathrm{~mm}$ chez les animaux traités au FGA + PMSG. Comme la décharge ovulante se produit I7 heures environ après le retrait de l'éponge (TESTART et KANN, I973), lorsque les animaux ont été traités par FGA + PMSG, il paraît exclu que sans traitement gonadotrope l'ovulation puisse se produire.

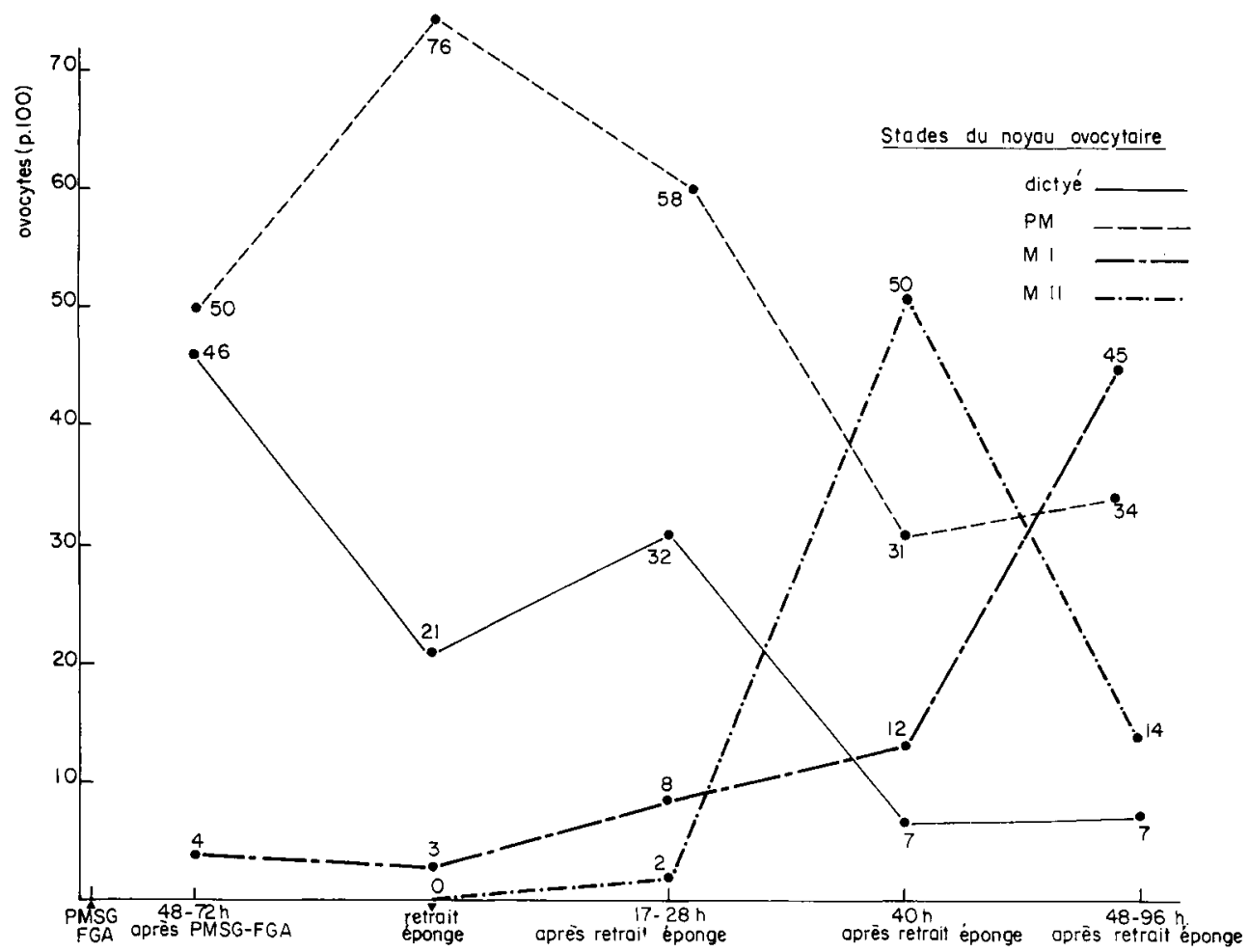

FIG. 2. - Fréquence relative des stades du noyau ovocytaire dans les follicules de 6 à $14 \mathrm{~mm}$

Si les images des chromosomes en première métaphase méïotique décrites par DonahuE (I968) chez la Souris et par Mandu (I963) chez le Rat peuvent être considérées comme caractéristiques pour tous les Mammifères, certains ovocytes en PM 
et même en MI où les chromosomes sont condensés et les cellules périovocytaires contractées, correspondraient à des ovocytes en dégénérescence. Les effectifs en MI (fig. 2) suivent une évolution qui n'a aucun rapport avec celle suivie par les effectifs en MII. La figure 2 révèle clairement qu'au moment où les effectifs en MII diminuent de $36 \mathrm{p}$. Io0, les effectifs en MI continuent à augmenter de 33 p. Ioo.

Ceci nous indique que peut-être seuls les ovocytes en MI, qui présentent l'image décrite par DONahuE (Ig68) peuvent passer en MIII. Ce passage se fait très rapidement. Les autres ovocytes en MI dont la chromatine est condensée, doivent s'arrêter dans cet état et dégénérer.

Rappelons que presque toute cette population ovocytaire se trouve incluse dans des follicules lutéinisés ou en voie de dégénérescence, donc ces follicules ne peuvent pas continuer leur évolution. Les résultats de NEAL et BAKER (I973) d'une part et ceux décrits dans le présent travail d'autre part, mettent en relief que l'un des états où s'arrête la méiose en cas d'atrésie est la métaphase I, spécialement lorsque l'ovocyte est inclus dans un follicule petit ou dégénéré.

En conclusion, il apparaît que le FGA joue un rôle favorable pour augmenter le stock des petits follicules normaux à antrum, mais que la gonadotropine exogène, tout en étant indispensable pour assurer une croissance folliculaire suffisante, pour permettre l'ovulation, précipite le phénomène d'atrésie.

Après la décharge ovulante, que l'ovulation se produise ou non, une atrésie générale frappe toutes les catégories de follicules à antrum.

\section{E. - Évolution de la taille des follicules pendant et après le traitement}

A partir de 1'examen des ovaires in toto (tabl. I) on constate que :

- 48 à 72 heures après le début du traitement sont présent en moyenne 35 follicules de 3 à $6 \mathrm{~mm}$ et 22 follicules de taille supérieure à $6 \mathrm{~mm}$;

- au moment du retrait de l'éponge, 96 heures après le début du traitement, 1'effectif folliculaire de la classe $3-6 \mathrm{~mm}$ a diminué de moitié ( $\mathrm{r} 7$ ), au profit de la classe de plus de $6 \mathrm{~mm}$ dont l'effectif est doublé (42) (tabl. I);

- dans les heures suivantes et jusqu'à 28 heures après le retrait de l'éponge, cette classe continue à s'enrichir (environ 70 follicules), alors que 1'effectif de la classe 3-6 $\mathrm{mm}$ ne change pas. Ceci indique que la classe $3-6 \mathrm{~mm}$ est continuellement alimentée à partir du pool de follicules de moins de $3 \mathrm{~mm}$.

Si l'on ne prend en compte que les follicules normaux à antrum, quelle que soit leur taille et contenant des ovocytes dictyés, leur nombre est de $\mathrm{r} 6$. Celui-ci est inférieur à celui des follicules normaux (27) qui contiennent des ovocytes en MI-MII au moment de l'ovulation. Ceci indique que les follicules qui ovulent ne sont pas nécessairement différenciés sur l'ovaire 48-72 heures après le début du traitement.

Le nombre de follicules normaux de $6 \mathrm{~mm}$ ( $\mathrm{I} 7$ ) contenant des ovocytes dictyés ou en prométaphase juste après la décharge ovulante ( $I_{7}$ à 28 heures après le retrait de l'éponge) est assez voisin du nombre de follicules normaux en MI et en MII au moment de l'ovulation (tab1. 4 et fig. 3). 


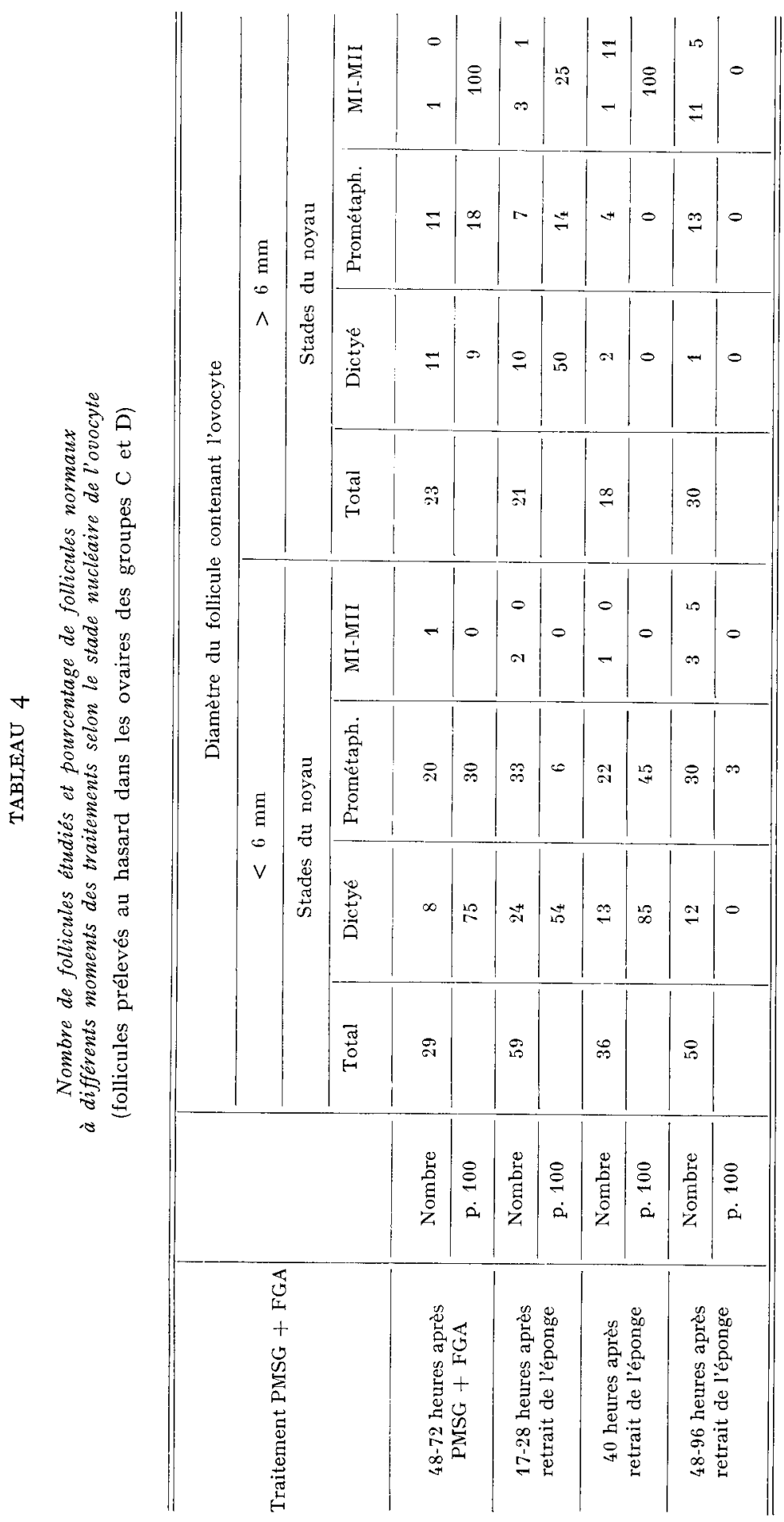




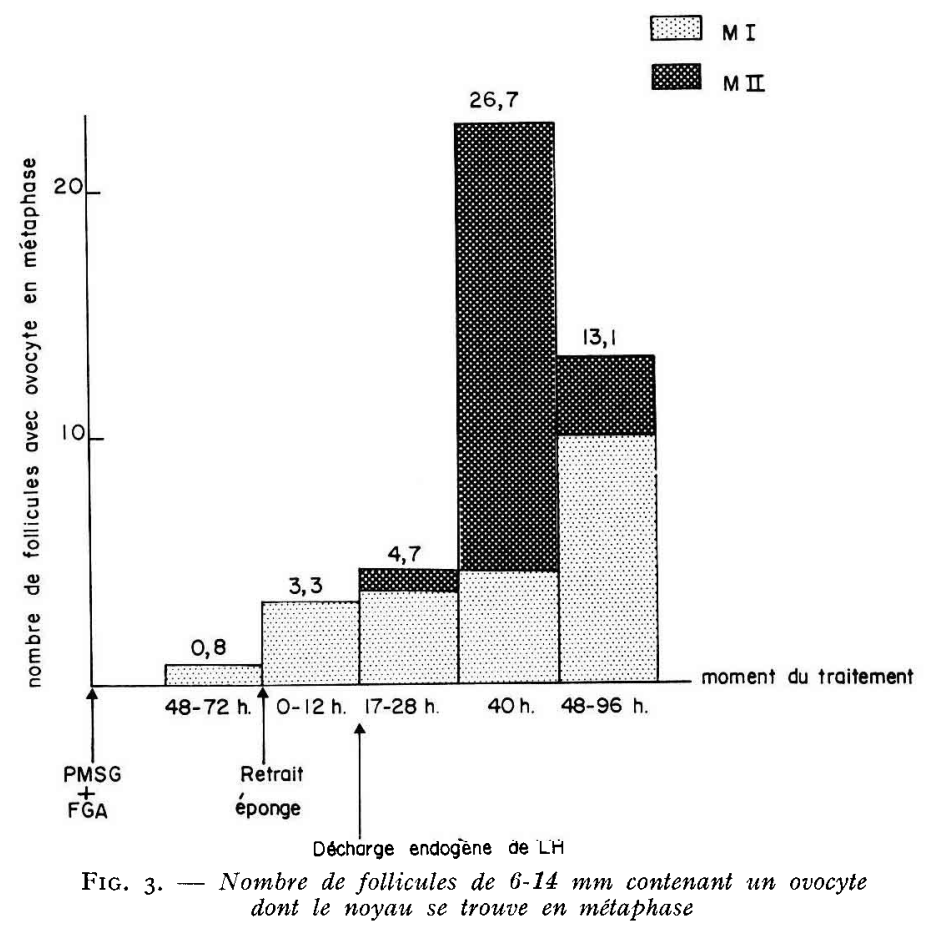

\section{F. - Taille des follicules préovulatoires et moment de l'ovulation}

La présence de la macula pellucida du stigma dans la partie du follicule qui fait saillie à la surface de l'ovaire permet de reconnaître les follicules préovulatoires 4o heures après le retrait de l'éponge. Ces follicules ont une taille comprise entre I 2 et $\mathrm{I} 4 \mathrm{~mm}$ de diamètre; ils se situent préférentiellement autour du hile ovarien. Cette taille correspond chez la Vache cyclique à celle du follicule préovulatoire 24 heures avant l'ovulation, c'est-à-dire au moment du pic de L.H (Marion et GIER, I97I).

Chez la Vache cyclique (Marion, Gier et Choudary, ig68; Marion et Gier, I97I), le follicule atteint I6 à I9 $\mathrm{mm}$ au moment de l'ovulation. Dans notre expérience chez le Veau traité avec PMSG + FGA, nous n'avons jamais observé de follicules de cette taille lorsque survient l'ovulation, 4o heures après le retrait de l'éponge, c'est-à-dire 23 heures après le pic maximal de $\mathrm{L} / \mathrm{H}$.

Sur les 8 veaux étudiés à ce moment, 3 avaient commencé à ovuler avec une moyenne de 5 ovulations. 48 heures plus tard, IOO p. Ioo de veaux ainsi traités avaient ovulé.

Reçu pour publication en avril 1974.

\section{REMERCIEMENTS}

Nous tenons à exprimer notre profonde reconnaissance à M. Thibault pour les précieux conseils qu'il nous a donnés tout au long de cette étude et la bienveillance qu'il nous a témoignée. 
Nous remercions la Direction générale de l'I. N. R. A. qui a mis à notre disposition les animaux et les installations expérimentales ainsi que M. LE Provost (LAthevet) qui nous a aimablement fourni le FGA.

\title{
SUMMARY
}

\author{
FOLLICULAR KINETICS AND OOCYTE MATURATION \\ IN THE CALF TREATED WITH FIUOROGESTONE ACETATE, AND PMSG
}

Follicular growth and resumption of meiosis is studied in fifty-two 3-4 month-old FFPN heifers following gonadotropic stimulation (I 800 IU of PMSG). Vaginal sponges impregnated with $60 \mathrm{mg} \mathrm{FGA}$ (fluorogestone acetate) are put in 49 heifers the same day as PMSG injection and left in place for 4 days.

The animals used are distributed in 4 groups : - Group A (controls) receiving no treatment; - Group B receiving only FGA during 4 days; - Groups C and D receiving PMSG plus progestagen. Those of group $\mathrm{C}$ are slaughtered $4^{8}$ and 72 hours after treatment is initiated, and those of group D when the sponge is removed (4 days after beginning of treatment), or up to 96 hours after the sponge is taken out.

After the slaughter of these animals, oocyte maturation is studied (nuclear transformation of the oocyte). The oocytes are obtained by aspiration of follicular fluid, or they are studied in relation to the follicle in which they are contained. In both cases they are fixed in Bouin-Hollande solution and stained with hematoxylin-eosine, trichrome of Masson and PAS. Normality or atresia of the follicles is described in this work.

In group A, only $35 \mathrm{p}$. I oo of the whole antral follicle population studied contains oocytes in dictyate stage. The size of these follicles is $0.72 \pm 0.4 \mathrm{~mm} ; 65 \mathrm{p}$. I 00 show signs of nuclear activation and their average size is $2.25 \pm 1.2 \mathrm{~mm}$. In the first group, only 36 p. Ioo of follicles are completely normal, but in the second group all show signs of atresia (table $3 a$, fig. I). In group B, 74 p. I oo of the whole antral follicle population studied (average diameter I.42 $\pm 0.25 \mathrm{~m}$ ) contains oocytes in dictyate stage. Of these, $62 \mathrm{p}$. Ioo are completely normal. The rest of the follicle population ( $26 \mathrm{p}$. I 00 ) contains oocytes having a nucleus which shows signs of activation (table $3 b$, fig. I).

In conclusion, we can say that FGA has a favorable action in preventing the evolution of follicle atresia, increasing the stock of little antral follicles, and avoiding the spontaneous resumption of meiosis.

In groups C and D, TESTART and KANN (I973) demonstrated that I5-20 hours after sponge removal, a LH peak occurs. This endogenic surge is responsible for the evolution of atresia and follicle luteinization. A study of nuclear activation of the oocyte shows that the favorable effect of FGA (when used alone) is counterbalanced by gonadotropic stimulation of the PMSG, and that the number of follicles which would be available to respond to an endogenic LH surge is reduced by PMSG.

Preovulatory follicles are recognized by the presence of the "macula pellucida", and by later histological study of the oocyte and the follicle in which it is contained.

The preovulatory follicle is found 40 hours after sponge removal; its diameter varies between I 2 and I4 $\mathrm{mm}$. The oocyte appears often in metaphase II.

\section{RÉFÉRENCES BIBLIOGRAPHIQUES}

Avery T. L., Fahning M. L., Graham G. F., I962. Investigations associated with the transplantation of bovin ova. J. Rep. Fert., 3, $212-217$.

Casida L. E., Meyer R. K., McShan W. H., Wisnicky W., i943. Effects of pituitary gonadotropins on the ovaries and the induction of superfecundity in cattle. Am. J. Vet. Res., 4, 76-94.

Choudary J.-B., Gier H. T., Marion G. B., ig68. Cyclic changes in bovine vesicular follicles. $J$. anim. Sci., 27, 468-47I.

DrsjaRdins C., Hafs H. D., I969. Maturation of bovine female genitalia from birth through puberty. J. anim. Sci., 28, 502-507. 
Dobson H., Hopkinson C. R. N., Word W. R., I973. Progesterone, I7- $\beta$-cestradiol and LH, in relation to ovulations in cows. Vet. Rec., 93, 76.

DonahuE P. R., I968. Maturation of the mouse oocyte in vitro. I. Sequence and timing of nuclear progression. J. Exp. Zool., 169, $237-250$.

Fox R. R., Cavanaugh J. L. Jr, ZarRow M. X., I964. Influence of age on the response of the immature rabbit ovary to PMS. Endocrinology, 75, 4II-4I6.

Gates A., Runner M. N., Jackson R. B., 1957. Influence of prepuberal age on number of ova that can be superovulated in the mouse. Anat. Rec., 123, 554 (Abstr.).

Hafez E. S. E., Sugie T., Hunt W. L., Ig63. Superovilation and related phenomena in the beef cow. J. Reprod. Fert., 5, $38 \mathrm{I}-388$.

HAFE\% E. S. E., I970. Superovulation and preservation of mammalian eggs. Acta Endoc*, 62, suppl. I40, $44 \mathrm{p}$.

Henricks D. M., Dickey J. F., Niswender G. D., I97o, Serum Luteinizing Hormone and Plasma Progesterone levels during the estrous cycle and early Pregnancy in cows. Biol. Reprod., 2, 346-35I.

Lineweaver J. A., Hafez E. S. E., I97o. Ovarian responses in gonadotropin-treated calves. Amer. J. Vet. Res., 31, 2157-2166.

Mande A. M., I963. Preovulatory changes in the oocyte of the adult Rat. Proc, roy. Soc., B, 158, I05-II8.

MARDEN W. G. R., 1952. The hormone control of ovulation in the calf. Endocrinology, 50, 456-46r.

Marden W. G. R., I953. The hormone control of ovulation in the calf. J. Agric. Sci., 43, $38 \mathrm{r}-406$.

Marion G. B., Gier H. T., Choudary J. B., r968. Micromorphology of the bovine ovarian follicular system. J. anim. Sci., 27, 45I-465.

Marion Cr. B., Gier H. T., I97I. Ovarian and uterine embryogenesis and morphology of the nonpregnant female mammal. J. Anim. Sci., 32, suppl. 1, 24-47.

Moss S., Wrenn T. R., Sykes J. F., r954. Some histological and histochemical observations of the bovine ovary during the estrous cycle. Anat. Rec., 120, 409-433.

MotтA P., i965. Sur l'ultrastructure des "Corps de Call et d'Exner " dans l'ovaire du Lapin. Z. Zellforsch., 68, 308-319.

Neal P., Baker T. G., 1973. Response of mouse ovaries in vivo and in organ culture to PMSG and HCG. I. Examination of critical time intervals. J. Reprod. Fertil., 33, 399-4 ro.

Onuma H., Hahn J., Maurer R. R., Foote R. H., ig69. Reported superovulation in calves. J. anim. Sci., 28, 634-637.

Onuma H., HahN J., Foote, R. H., I97o. Factors affecting superovulation, fertilization and recovery of superovulated ova in prepubertal cattle. J. Reprod. Fert., 21, I I9-1 26.

Picon L., 1956. Sur le rôle de l'âge dans la sensibilité de l'ovaire à l'hormone gonadotrope chez le Rat. Arch. Anat. Micr. Morph. Exp., 45, 311-341.

Priedkalns J., Weber F. A., Zkmjanis R., 1968. Qualitative and quantitative morphological studies of the cells of the membrana granulosa, theca interna and corpus luteum of the bovine ovary. Z. Zellforsch., 85, 501-520.

RAJAKoski E., I960. The ovarian follicular system in sexually mature heifers with special reference to seasonal, cyclical and left-right variations. Acta endocr., suppl., 52, r-68.

Rowson L. E., I95I. Methods of inducing multiple ovulation in cattle. J. Endocr., 7, 260.

Seidel G. E., Larson L. L., Foote R. H., i97I. Effects of age and gonadotropin treatment on superovulation in the calf. J. anim. Sci., 33, 6r7-622.

Testart J., I972 a. Synchronisation des ovulations induites chez le Veau femelle impubère. VII intern. Kongr. für tierische Fort flanzung, München, I972, 1, 493-498.

Testart J., I972 $b$. Réponse folliculaire du Veau femelle impubère à divers traitements de gonadotropine sérique associée ou non à un progestagène. Ann. Biol. anim. Bioch. Biophys., 12, 397-409.

Testart J., Arrau J., I973. Maturation ovocytaire après stimulation folliculaire chez la Génisse impubère, Ann. Biol. anim. Bioch. Biophys., 13, no hors série, r57-165.

Testart J., Kann G., I973. Taux plasmatique de $\mathrm{LH}$ chez la Génisse impubère soumise à un traitement de superovulation. C. R. Acad. Sci., Paris, D, 277, I I8I-1 I84.

Thibault C., I949. L'œuf des Mammifères. Son développement parthénogénétique. Thèse Dr Sci., Fac. Sci. Paris. Ann. Sci. Nat. Zool., 11, I 8 I-1 I84.

ZarRow M. X., WiLSoN E. D., I96r. The influence of age on superovulation in the immature rat and mouse. Endocrinology, 69, 85 I-855. 


\section{PLANCHE I}

\section{Photo I}

Aspect morphologique de l'appareil génital du Veau témoin : C : col de l'utérus

$\mathrm{U}$ : utérus

$\mathrm{T}$ : trompe de Fallope

$\mathrm{O}$ : ovaire

\section{Рното 2}

Aspect des ovaires du Veau au moment du retrait de l'éponge imprégnée avec FGA.

\section{Рното 3}

Aspects des ovaires du Veau, 28 heures après le retrait de l'éponge.

\section{Photo 4}

Aspect des ovaires du Veau, 48 heures après le retrait de l'éponge (les flèches indiquent les points d'ovulation). 

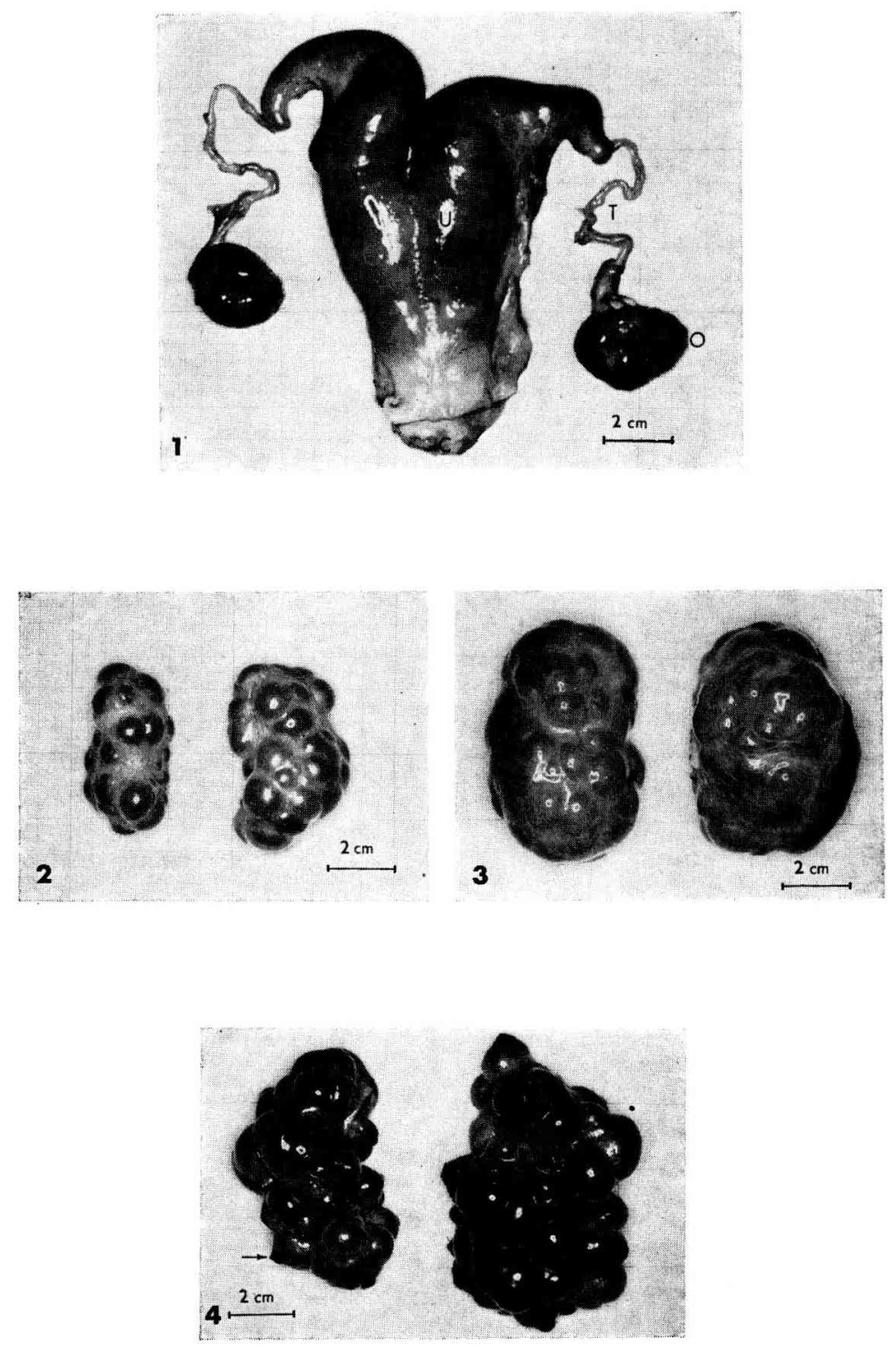


\section{PLANCHE II}

\section{Рното 5}

Paroi folliculaire avec le cumulus oophorus (CO) renfermant l'ovocyte ( $X$ IOO).

\section{Рното 6}

Aspect de cet ovocyte à un plus fort grossissement ( $\times 500)$.

$$
\begin{aligned}
& \mathrm{N}: \text { noyau } \\
& \mathrm{n}: \text { nucléole }
\end{aligned}
$$

\section{Pнoto 7}

Paroi du même follicule $(\times 300)$

$G$ : granulosa normale

' $\mathrm{T}$ : thèques

(la flèche indique la membrane basale)

\section{Photo 8}

Paroi du grand follicule lutéinisé ( 72 heures après le retrait de l'éponge) ( $\times 80$ ) (la flèche indique l'ovocyte).

\section{Рното 9}

Ovocyte du même follicule, entouré de cellules lutéinisées $(\times 320)$

$$
\begin{aligned}
& \mathrm{O}: \text { ovocyte } \\
& \mathrm{CL} \text { : cellules du cumulus lutéinisées }
\end{aligned}
$$

\section{Рното го}

Paroi du même follicule $(\times 320)$. Observer la lutéinisation des cellules de la granulosa et des thèques (GTL). 

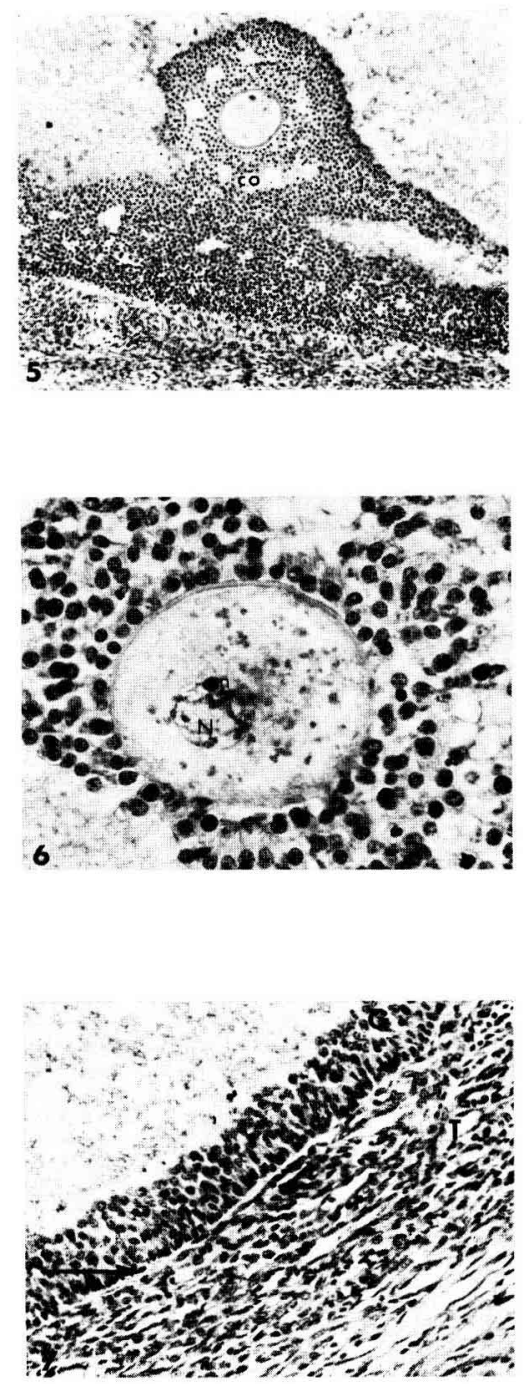
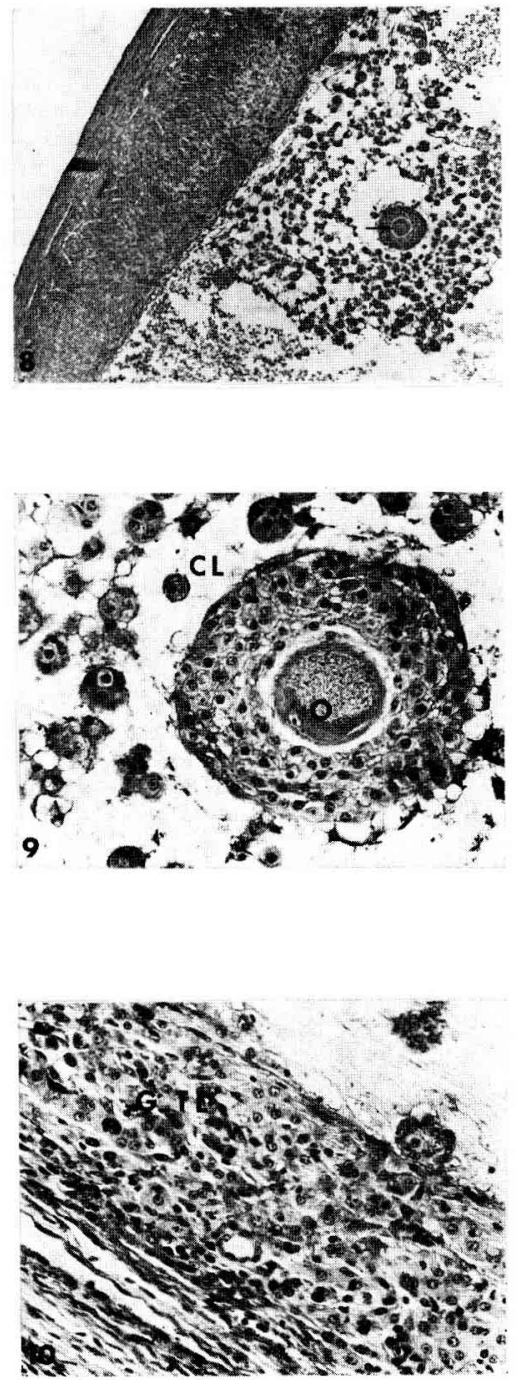


\section{PLANCHE 3}

Follicule préovulatoire de de Graaf

Ph. 11 a : Vue d'ensemble de la paroi folliculaire et du cumulus $(\times 32)$.

CO : Cumulus oophorus (la flèche montre l'ovocyte).

Ph. $11 \mathrm{~b}$ : A un plus fort grossissement, l'ovocyte en M II $(\times 200)$ montre la plaque métaphasique et le premier globule polaire (gp).

Ph. 12 : Paroi du même follicule $(\times 200)$.

La flèche (l.f.) indique la présence de liquide folliculaire secondaire.

V.S. : Vaisseau sanguin.

Ph. 13 : Avec un grossissement supérieur $(\times 320$ et 800$)$ on peut distinguer les tissus thécal et granulosique (TG). On remarque, en outre, la disparition de la membrane basale. (La flèche indique deux cellules de cet ensemble déjà lutéinisées). 

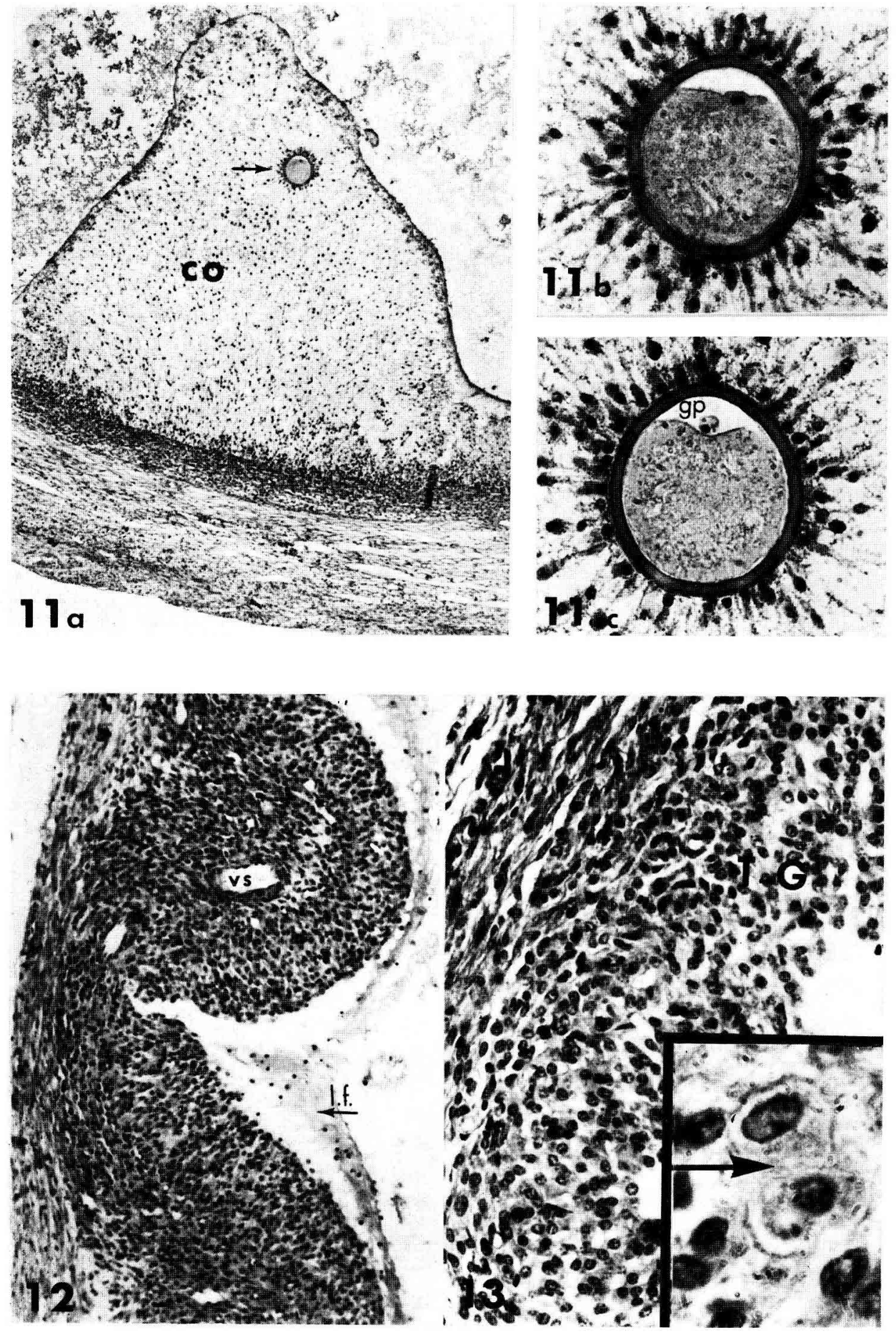